\title{
An extensive comparison of species-abundance distribution models
}

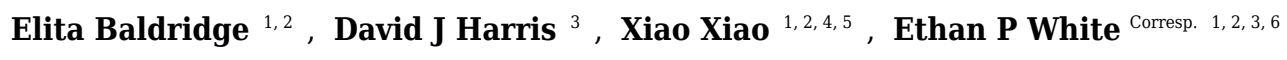

1 Department of Biology, Utah State University, Logan, Utah, United States

2 Ecology Center, Utah State University, Logan, Utah, United States

3 Department of Wildlife Ecology and Conservation, University of Florida, Gainesville, FL, United States

${ }^{4}$ School of Biology and Ecology and Senator George J. Mitchell Center for Sustainability Solutions, University of Maine, Orono, Maine, United States

5 Mitchell Center for Sustainability Solutions, University of Maine, Orono, Maine, United States

${ }^{6}$ Informatics Institute, University of Florida, Gainesville, FL, United States

Corresponding Author: Ethan P White

Email address: ethan@weecology.org

A number of different models have been proposed as descriptions of the speciesabundance distribution (SAD). Most evaluations of these models use only one or two models, focus only a single ecosystem or taxonomic group, or fail to use appropriate statistical methods. We use likelihood and AIC to compare the fit of four of the most widely used models to data on over 16,000 communities from a diverse array of taxonomic groups and ecosystems. Across all datasets combined the log-series, Poisson lognormal, and negative binomial all yield similar overall fits to the data. Therefore, when correcting for differences in the number of parameters the log-series generally provides the best fit to data. Within individual datasets some other distributions performed nearly as well as the log-series even after correcting for the number of parameters. The Zipf distribution is generally a poor characterization of the SAD. 


\section{An extensive comparison of species-abundance distribution models}

2 Elita Baldridge ${ }^{1,2}$, David J. Harris ${ }^{3}$, Xiao Xiao ${ }^{1,2,4,5}$, Ethan P. White ${ }^{*}, 1,2,3,6$

$3 \quad{ }^{1}$ Department of Biology, Utah State University, Logan, Utah, USA

$4 \quad 2$ Ecology Center, Utah State University, Logan, Utah, USA

$5{ }^{3}$ Department of Wildlife Ecology \& Conservation, University of Florida, Gainesville, Florida,

6 USA

$7 \quad{ }^{4}$ School of Biology and Ecology, University of Maine, Orono, Maine, USA

$8 \quad{ }^{5}$ Mitchell Center for Sustainability Solutions, University of Maine, Orono, Maine, USA

$9 \quad{ }^{6}$ Informatics Institute, University of Florida, Gainesville, Florida, USA 


\section{Abstract}

12 A number of different models have been proposed as descriptions of the species-

13 abundance distribution (SAD). Most evaluations of these models use only one or two

14 models, focus only a single ecosystem or taxonomic group, or fail to use appropriate

15 statistical methods. We use likelihood and AIC to compare the fit of four of the most widely

16 used models to data on over 16,000 communities from a diverse array of taxonomic groups

17 and ecosystems. Across all datasets combined the log-series, Poisson lognormal, and

18 negative binomial all yield similar overall fits to the data. Therefore, when correcting for

19 differences in the number of parameters the log-series generally provides the best fit to

20 data. Within individual datasets some other distributions performed nearly as well as the

21 log-series even after correcting for the number of parameters. The Zipf distribution is

22 generally a poor characterization of the SAD.

\section{Introduction}

24 The species abundance distribution (SAD) describes the full distribution of commonness

25 and rarity in ecological systems. It is one of the most fundamental and ubiquitous patterns

26 in ecology, and exhibits a consistent general form with many rare species and few

27 abundant species occurring within a community. The SAD is one of the most widely studied

28 patterns in ecology, leading to a proliferation of models that attempt to characterize the

29 shape of the distribution and identify potential mechanisms for the pattern (see McGill et

30 al. 2007 for a recent review of SADs). These models range from arbitrary distributions that

31 are chosen based on providing a good fit to the data (Fisher et al. 1943), to distributions 
32 chosen based on the most likely states of generic random systems (Frank 2011, Harte 33 2011, Locey and White 2013), to models based more directly on ecological processes 34 (Tokeshi 1993, Hubbell 2001, Volkov et al. 2003, Alroy 2015).

35 Which model or models provide the best fit to the data, and the resulting implications for 36 the processes structuring ecological systems, is an active area of research (e.g., McGill 2003, 37 Volkov et al. 2003, Ulrich et al. 2010, White et al. 2012, Connolly et al. 2014). However, 38 most comparisons of the different models: 1) use only a small subset of available models 39 (typically two; e.g., McGill 2003, Volkov et al. 2003, White et al. 2012, Connolly et al. 2014); 40 2) focus on a single ecosystem or taxonomic group (e.g., McGill 2003, Volkov et al. 2003); or 41 3) fail to use the most appropriate statistical methods (e.g., Ulrich et al. 2010, see Matthews 42 and Whittaker 2014 for discussion of best statistical methods for fitting SADs). This makes 43 it difficult to draw general conclusions about which, if any, models provide the best 44 empirical fit to species abundance distributions.

45 Here, we evaluate the performance of four of the most widely used models for the species 46 abundance distribution using likelihood-based model selection on data from 16,209 47 communities and nine major taxonomic groups. This includes data from terrestrial, aquatic, 48 and marine ecosystems representing roughly 50 million individual organisms in total. 


\section{Methods}

50

51

52

53

54

55

56

57

58

\section{Data}

We compiled data from citizen science projects, government surveys, and literature mining to produce a dataset with 16,209 communities, from nine taxonomic groups, representing nearly 50 million individual terrestrial, aquatic, and marine organisms. Data for trees, birds, butterflies and mammals was compiled by White et al. (2012) from six data sources: the US Forest Service Forest Inventory and Analysis (FIA; USDA Forest Service 2010), the North American Butterfly Association's North American Butterfly Count (NABC; North American Butterfly Assoc. 2009), the Mammal Community Database (MCDB; Thibault et al. 2011), Alwyn Gentry's Forest Transect Data Set (Gentry; Phillips and Miller 2002), the Audubon Society Christmas Bird Count (CBC; National Audubon Society 2002), and the US Geological Survey's North American Breeding Bird Survey (BBS; Pardieck et al. 2014) (see Table 1 for details). The publicly available datasets (FIA, MCDB, Gentry, and BBS) were acquired using the EcoData Retriever (http://ecodataretriever.org; Morris and White 2013). Details of the treatment of these datasets can be found in Appendix A of White et al. (2012), but in general data were analyzed at the level of the site defined in the dataset and a single year of data was selected for each site. We modified the data slightly by removing sites 102 and 179 from the Gentry data due to issues with decimal abundances appearing in raw data due to either data entry or data structure errors. Data on Actinopterygii, Reptilia, Coleoptera, Arachnida, and Amphibia, were mined from literature by Baldridge and are publicly available (Baldridge 2013) (see Table 1 for details). These data were collected at the level of the site defined in the publication if raw data were available at that 
71 scale, and at the scale of the entire study otherwise. Time scales of collection for this data

72 depended on the study but was typically one or a few years. All data sources used in the

73 analysis a samples (or censuses) of a taxonomic assemblage, where all individuals of any

74 species seen are recorded. Abundances in the compiled datasets were counts of individuals.

75 Table 1: Details of datasets used to evaluate the form of the species abundance distribution.

76 Datasets marked as Private were obtained through data requests to the providers.

Total

Dataset Dataset code Availability sites Citation

Breeding Bird $\quad$ BBS $\quad$ Public $2769 \quad$ Pardieck et al. (2014)

Survey

\begin{tabular}{llll}
\hline Christmas & CBC & Private & $1999 \quad$ National Audubon Society (2002)
\end{tabular}

Bird Count

\begin{tabular}{llll}
\hline Gentry's $\quad$ Gentry & Public & $220 \quad$ Phillips and Miller (2002)
\end{tabular}

Forest

Transects

\begin{tabular}{|c|c|}
\hline Forest & FIA \\
\hline
\end{tabular}

Inventory

Analysis

Mammal MCDB $\quad$ Public $103 \quad$ Thibault et al. (2011)

Community

Datatbase 


\begin{tabular}{lllll}
\hline NA Butterfly & NABA & Private & 400 & North American Butterfly Assoc. \\
Count & & & & $(2009)$ \\
\hline Actinopterygii & Actinopterygii & Public & 161 & Baldridge (2013) \\
\hline Reptilia & Reptilia & Public & 129 & Baldridge (2013) \\
\hline Amphibia & Amphibia & Public & 43 & Baldridge (2013) \\
\hline Coleoptera & Coleoptera & Public & 5 & Baldridge (2013) \\
\hline Arachnida & Arachnida & Public & 25 & Baldridge (2013) \\
\hline
\end{tabular}

79 We selected models for analysis based on four criteria. First, since the majority of species

80 abundance distributions (SADs) are constructed using counts of individuals (for discussion

81 of alternative approaches see McGill et al. 2007 and @morlon2009) we selected models

82 with discrete distributions (i.e., those that only have non-zero probabilities for positive

83 integer values of abundance). Second, in order to use best practices for comparing species

84 abundance distributions we selected models with analytically defined probability mass

85 functions that allow the calculation of likelihoods (see details in Analysis). Third, McGill et

86 al. (2007) classified species abundance distribution models into five different families:

87 purely statistical, branching process, population dynamics, niche partitioning, and spatial

88 distribution of individuals. We evaluated models from each of these families, with some

89 models having been derived from more than one family of processes. Finally, we selected

90 models that have been widely used in the ecological literature. Based on these criteria we 
91 evaluated the log-series, the Poisson lognormal, the negative binomial, and the Zipf

92 distributions. All distributions were defined to be capable of having non-zero probability at

93 integer values from 1 to infinity.

94 The log-series is one of the first distributions used to describe the SAD, being derived as a

95 purely statistical distribution by Fisher (1943). It has since been derived as the result of

96 ecological processes, the metacommunity SAD for ecological neutral theory (Hubbell 2001,

97 Volkov et al. 2003), and several different maximum entropy models (Pueyo et al. 2007,

98 Harte et al. 2008).

99 The lognormal is one of the most commonly used distributions for describing the SAD

100 (McGill 2003) and has been derived as a null form of the distribution resulting from the

101 central limit theorem (May 1975), population dynamics (Engen and Lande 1996), and

102 niche partitioning (Sugihara 1980). We use the Poisson lognormal because it is a discrete

103 form of the distribution appropriate for fitting discrete abundance data (Bulmer 1974).

104 The negative binomial (which can be derived as a Gamma-distributed mixture of Poisson

105 distributions) provides a good characterization of the SAD predictions for several different

106 ecological neutral models for the purposes of model selection (Connolly et al. 2014). We

107 use it to represent neutral models as a class.

108 The Zipf (or power law) distribution was derived based on both branching processes and

109 as the outcome of the McGill and Collin's (2003) spatial model. It was one of the best fitting

110 distributions in a recent meta-analysis of SADs (Ulrich et al. 2010). We use the discrete

111 form of the distribution which is appropriate for fitting discrete abundance data (White et

112 al. 2008). 
113 Figure 1 shows three example sites with the empirical distribution and associated models

114 fit to the data Zipf distributions tend to predict the most rare species followed by the log-

115 series, the negative binomial, and Poisson lognormal.
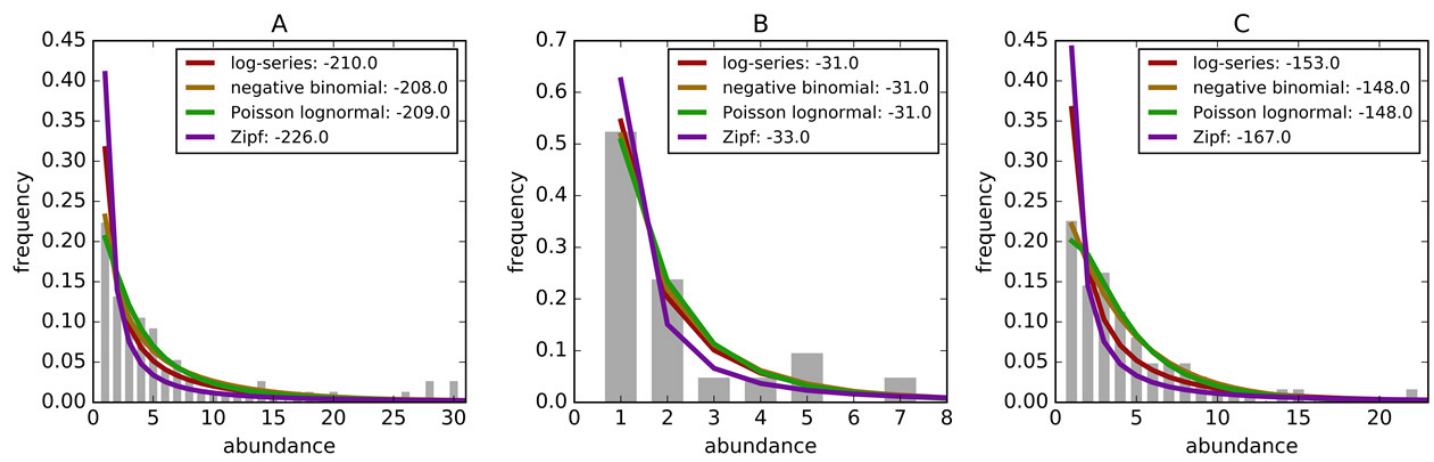

116

117 Example species-abundance distributions including the empirical distributions (grey bars)

118 and the best fitting log-series (black line), negative binomial (green line), Poisson lognormal

119 (red line), and Zipf (purple line). Distributions are for (a) Breeding Bird Survey - Route 36 in

120 New York, (b) Forest Inventory and Analysis - Unit 4, County 57, Plot 12 in Alabama, and (c)

121 Gentry - Araracuara High Campina site in Colombia. Log-likelihoods of the models are

122 included in parenthesis in the legend

123 Analysis

124 Following current best practices for fitting distributions to data and evaluating their fit, we

125 used maximum likelihood estimation to fit models to the data (Clark et al. 1999, Newman

126 2005, White et al. 2008) and likelihood-based model selection to compare the fits of the

127 different models (Burnham and Anderson 2002, Edwards et al. 2007). This approach has

128 recently been affirmed as best practice for species abundance distributions (Connolly et al.

129 2014, Matthews and Whittaker 2014). This requires that likelihoods for the models can be 
130 solved for and therefore we excluded models that lack probability mass functions and

131 associated likelihoods. While methods have been proposed for comparing models without

132 probability mass functions in this context (Alroy 2015), these methods have not been

133 evaluated to determine how well they perform compared to the widely accepted

134 likelihood-based approaches.

135 For model comparison we used corrected Akaike Information Criterion (AICc) weights to

136 compare the fits of models while correcting for differences in the number of parameters

137 and appropriately handling the small sample sizes (i.e., numbers of species) in some

138 communities (Burnham and Anderson 2002). The Poisson lognormal and the negative

139 binomial each have two fitted parameters, while the log-series distribution and the Zipf

140 distributions have one fitted parameter each. The model with the greatest AICc weight in

141 each community was considered to be the best fitting model for that community. We also

142 assessed the full distribution of AICc weights to evaluate the similarity of the fits of the 143 different models.

144 In addition to evaluating AICc of each model, we also examined the log-likelihood values of

145 the models directly. We did this to assess the fit of the model while ignoring corrections for

146 the number of parameters and the influence of similarities to other models in the set of

147 candidate models. This also allows us to make more direct comparisons to previous

148 analyses that have not corrected for the number of parameters (i.e., Ulrich et al. 2010, Alroy

149 2015)

150 Model fitting, log-likelihood, and AICc calculations were performed using Python (Van

151 Rossum and Drake 2011) and R (R Core Team 2015). Python packages used for analysis 
152 include numpy (Oliphant 2007, Van Der Walt et al. 2011), matplotlib (Hunter and others

153 2007), sqlalchemy (Bayer 2014), pandas (McKinney and others 2010), macroecotools (Xiao

154 et al. 2016), retriever (Morris and White 2013), R packages used for analysis include

155 ggplot2 (Wickham 2009), magrittr(Bache and Wickham 2014), tidyr (Wickham 2016),

156 dplyr (Wickham and Francois 2016). All of the code and all of the publicly available data

157 necessary to replicate these analyses is available at https://github.com/weecology/sad-

158 comparison and archived on Zenodo (Baldridge et al. 2016). The CBC datasets and NABA

159 datasets are not publicly available and therefore are not included.

160 Results

161 Across all datasets, the negative binomial and Poisson lognormal distributions had very

162 similar average log-likelihoods (within 0.01 of one another; Figure 2). The log-likelihoods

163 for each of these distributions averaged 0.8 units higher than for the log-series distribution

164 and 5 units higher than for the Zipf distribution (corresponding to likelihoods that were

165 twice as high and 140 times as high, respectively). 


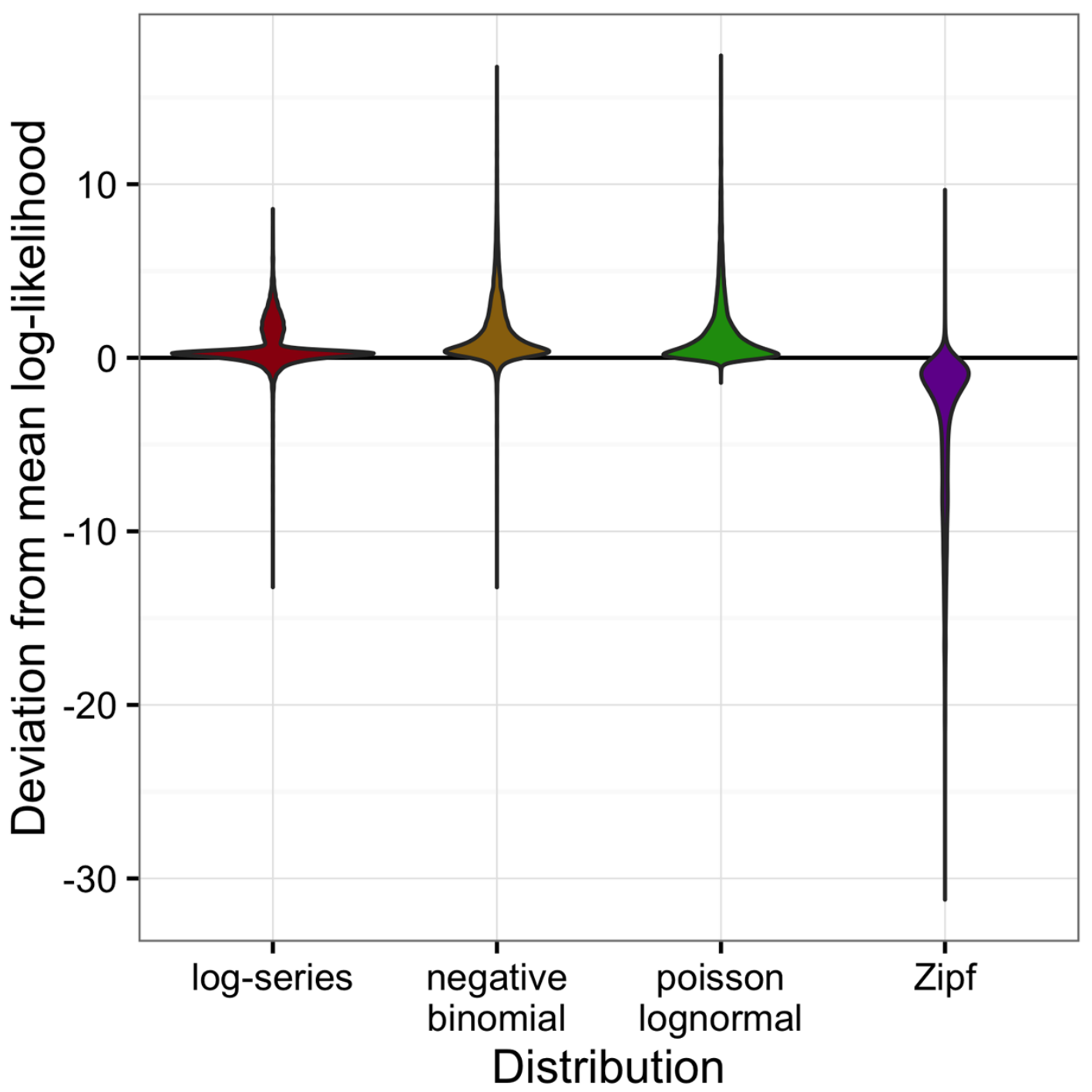

166

167 Violin plots of the deviation from the mean log-likelihood for each site for all datasets

168 combined. Positive values indicate that the model fits better than the average fit across the 169 four models.

170 Although the negative binomial and Poisson lognormal distributions matched the data

171 most closely, the likelihood provides a biased estimate of these distributions' ability to

172 generalize to unobserved species. AICc approximately removes this bias by penalizing

173 models with more degrees of freedom (e.g. the negative binomial and Poisson lognormal 
174 distributions, which have two free parameters instead of one like the log-series and Zipf

175 distributions). After applying this penalty, the log-series distribution would be expected to

176 make the best predictions for $69.2 \%$ of the sites. The Poisson lognormal and negative

177 binomial distributions were each preferred in about $12 \%$ of the sites, and the Zipf

178 distribution was preferred least often (6.0\% of sites; Figure 3).

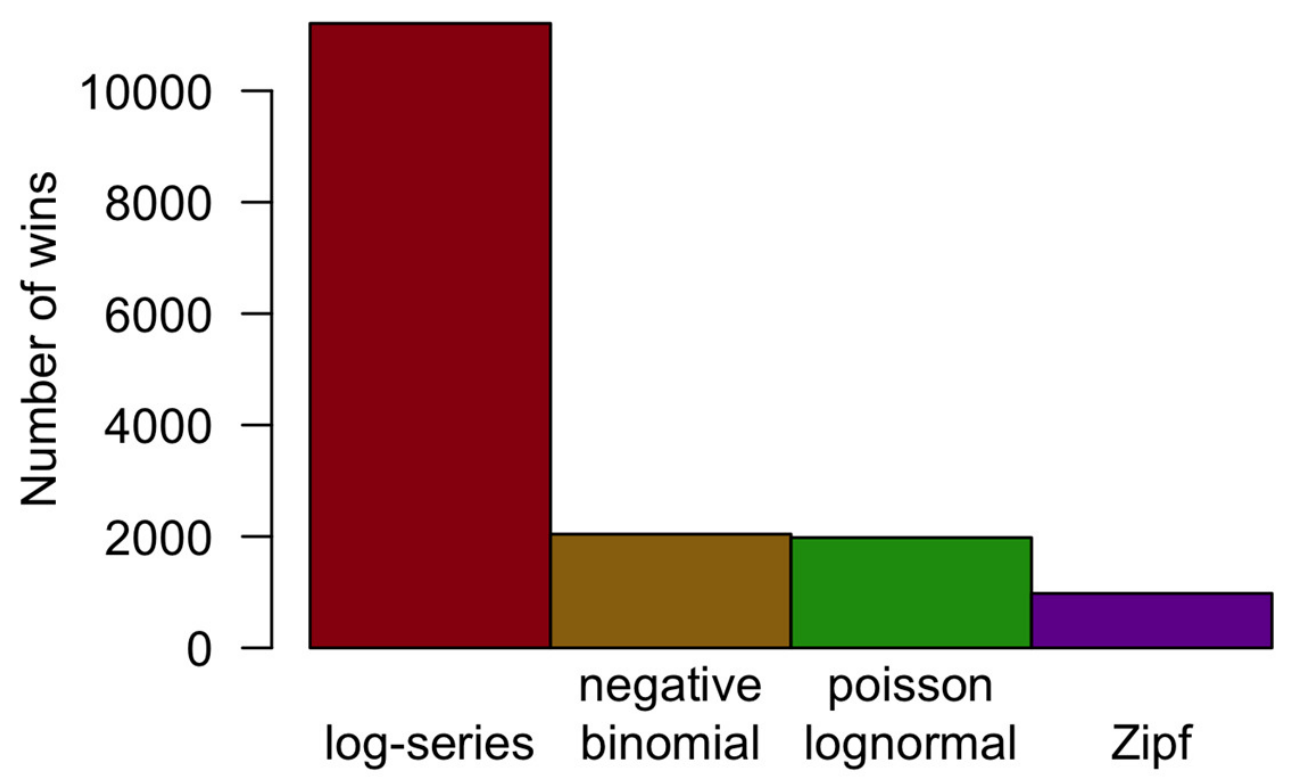

179

\section{Species abundance distribution}

Number of cases in which each model provided the best fit to the data based on AICc for all

datasets combined.

182 Across all datasets and taxonomic groups, the log-series distribution had the highest AICc

183 weights more often than any other model. The negative binomial performed well for BBS,

184 but was almost never the best fitting model for plants (FIA and Gentry), butterflies (NABA), 
185 Acintopterygii, or Coleoptera. The Poisson lognormal performed well for the bird datasets

186 (BBS and CBC) and the Gentry tree data, but was almost never best in the FIA and

187 Coleoptera datasets (Figure 4). The Zipf distribution only performed consistently well for

188 Arachnida. Because datasets differ in both taxonomic groups and sampling methods care

189 should be taken in interpreting these differences.

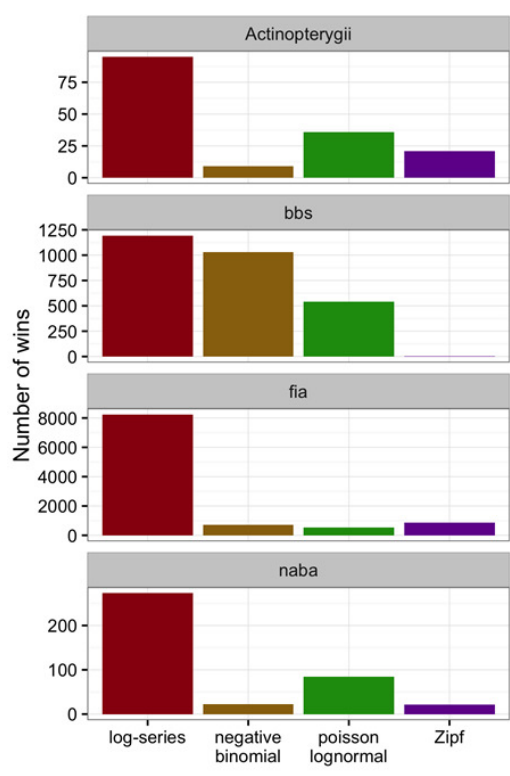

191 Number of cases in which each model provided the best fit to the data based on AICc for each

192 dataset separately.

193 The full distribution of AICc weights shows separation among models (Figure 5). Although

194 the log-series distribution had the best AICc score much more often than the other models,

195 its lead was never decisive: across all 16,209 sites, it never had more than about $75 \%$ of the

196 AICc weight (Figure 5). Most of the remaining weight was assigned to the negative binomial

197 and Poisson lognormal distributions (each of which usually had at least $12-15 \%$ of the 
198 weight but was occasionally favored very strongly). The Zipf distribution showed a strong 199 mode near zero, and usually had less than $7 \%$ of the weight.

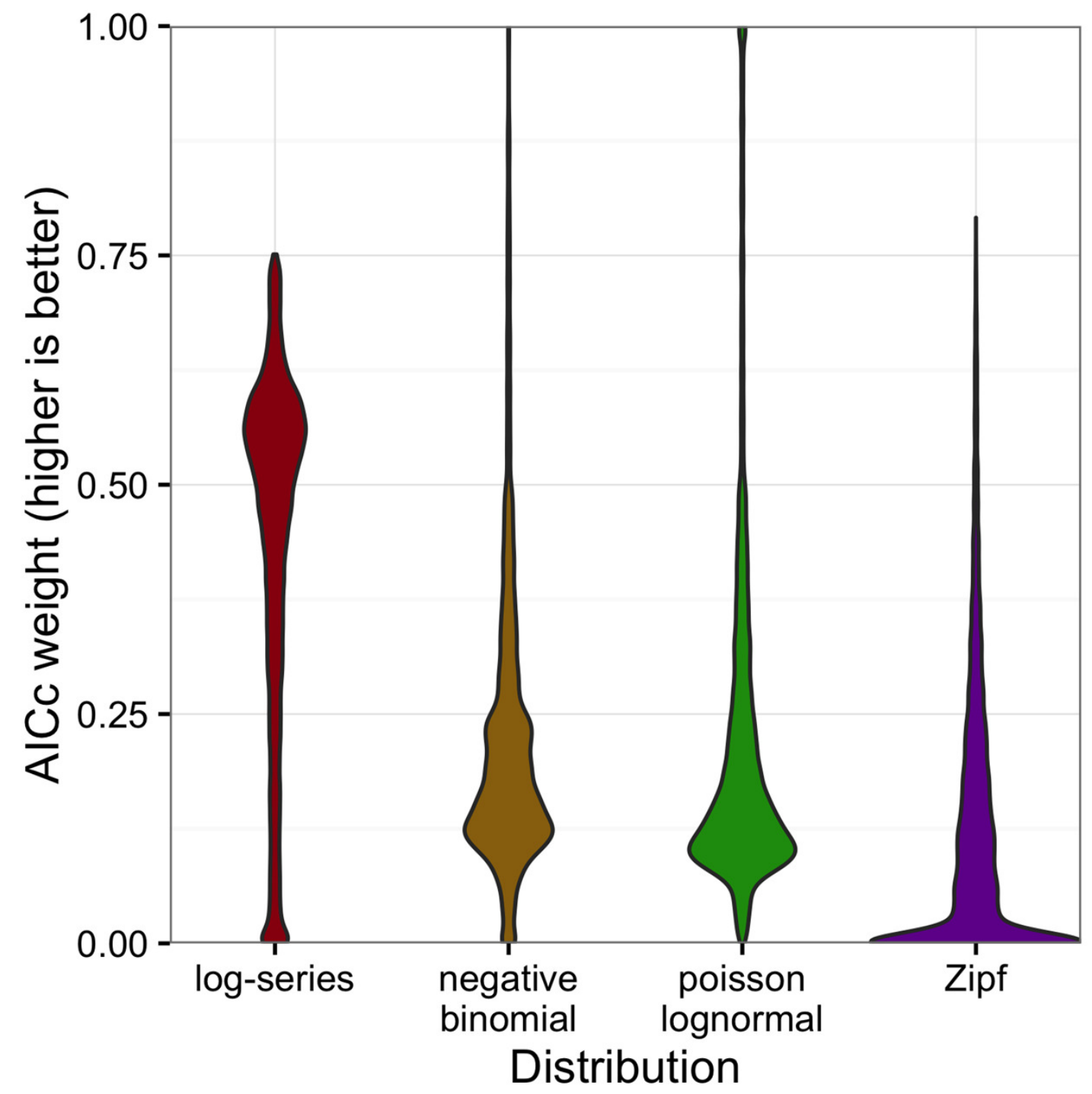

201 Violin plots of the AICc weights for each model. Weights indicate the probability that the 202 model is the best model for the data 


\section{Discussion}

204 Our extensive comparison of different models for the species abundance distribution (SAD)

205 using rigorous statistical methods demonstrates that several of the most popular existing

206 models provide equivalently good absolute fits to empirical data. Log-series, negative

207 binomial, and Poisson lognormal all had model relative likelihoods between 0.25 and 0.5

208 suggesting that the three distributions provide roughly equivalent fits in most cases, but

209 with the two-parameter model performing slightly better on average. Because the log-

210 series has only a single parameter but fits the data almost as well as the two-parameter

211 models, the log-series performed better in AICc-based model selection, which penalizes

212 model complexity. These results differ from two other recent analyses of large numbers of

213 species abundance distributions (Ulrich et al. 2010, Connolly et al. 2014) and are generally

214 consistent with a third recent analysis (Alroy 2015).

215 Ulrich et al. (2010) analyzed 500 SADs and found support for three major forms of the

216 SAD that changed depending on whether the community had been fully censused or not.

217 They found that "fully censused" communities were best fit by the lognormal, and

218 "incompletely sampled" communities, best fit by the Zipf and log-series (Ulrich et al. 2010).

219 In contrast we find effectively no support for the Zipf across ecosystems and taxonomic

220 groups, including a number of datasets that are incompletely sampled. Our AICc value

221 results also do not support the conclusion that the lognormal outperforms the log-series in

222 fully censused communities. The Gentry and FIA forest inventories both involve large

223 stationary organisms and were collected with the goal of including all trees above a certain

224 stem diameter. Therefore, above the minimum stem diameter, they are as close to fully 
225 censused communities as is typically possible. In these communities the log-series provides

226 the best fit to the data most frequently. The discrepancy between our results and those

227 found in (Ulrich et al. 2010) may be due to: 1) their use of binning and fitting curves to rank

228 abundance plots, which deviates from the likelihood-based best practices (Matthews and

229 Whittaker 2014) used in this paper; 2) the statistical methods they use to identify

230 communities as "fully censused", which tend to exclude communities with large numbers of

231 singletons that would be better fit by distributions like the log-series; 3) the use of the

232 continuous lognormal instead of the Poisson lognormal; 4) the fact that our censused

233 communities are also a different taxonomic group from our sampled communities, making

234 it difficult to distinguish between taxonomic and sampling differences.

235 Connolly et al. (2014) use likelihood-based methods to compare the negative binomial

236 distribution (which they call the Poisson gamma) to the Poisson lognormal for a large

237 number of marine communities. They found that the Poisson lognormal provides a

238 substantially better fit than the negative binomial to empirical data and that the negative-

239 binomial provides a better fit to communities simulated using neutral models. They

240 conclude that these analyses of the SAD demonstrate that marine communities are

241 structured by non-neutral processes. Our analysis differs from that in Connolly et al. (2014)

242 in that they aggregate communities at larger spatial scales than those sampled and find the

243 strongest results at large spatial scales. This may explain the difference between the two

244 analyses or there may be differences between the terrestrial systems analyzed here and the

245 marine systems analyzed by Connolly et al. (2014). The explanation for these differences is

246 being explored elsewhere (Connolly et al. unpublished data). 
247 Alroy (2015) compared the fits of the lognormal, log-series, Zipf, geometric series, broken

248 stick, and a new model dubbed the "double geometric", to over 1000 terrestrial community

249 datasets assembled from the literature. To incorporate the geometric series, broken stick,

250 and the double geometric, this research used non-standard methods for evaluating the fits

251 of the models to the data, however the results were generally consistent with those

252 presented here. The central Kullback-Leibler divergence statistics results showed that: 1)

253 the Zipf, geometric series, and broken stick all perform consistently worse than the other

254 distributions; 2) the double geometric, log-series, and lognormal all provide the best

255 overall fit for at least one taxonomic group; and 3) the lognormal and double geometric fit

256 the data equivalently well and slightly better than the log-series when not controlling for

257 differences in the number of parameters (Alroy's tables S1, S2, and S3). Penalizing the two-

258 parameter models (lognormal and double geometric) for their complexity, as we do here

259 with AICc, would likewise improve the relative performance of the log-series distribution.

260 In combination, the results of these three papers suggest that in general the Zipf is a poor

261 characterization of species-abundance distributions and that both the log-series and

262 lognormal distributions provide reasonable fits in many cases. Differences in the

263 performance of the log-series, lognormal, double geometric, and negative binomial, appear

264 to be more minor. How these differences relate to differences in intensity of sampling,

265 spatial scale, taxonomy, and ecosystem type (marine vs. terrestrial) remain open questions.

266 Our analyses suggest that controlling for the number of parameters makes the log-series a

267 slightly better fitting model, at least in the terrestrial systems we studied. Neither of the

268 other papers that include the log-series (Ulrich et al. 2010, Alroy 2015) make this 
269 correction and both show that it is still a reasonably competitive model even against those

270 with more parameters.

271 The relatively similar fit of several commonly used distributions emphasizes the challenge

272 of inferring the processes operating in ecological systems from the form of the abundance

273 distribution. It is already well established that models based on different processes can

274 yield equivalent models of the SAD, i.e., they predict distributions of exactly the same form

275 (Cohen 1968, Boswell and Patil 1971, Pielou 1975, McGill et al. 2007). To the extent that

276 SADs are determined by random statistical processes, one might expect the observed

277 distributions to be compatible with a wide variety of different process-based and process-

278 free models (Frank 2009, 2011, Locey and White 2013). Regardless of the underlying

279 reason that the models performed similarly, our results indicate that the SAD usually does

280 not contain sufficient information to distinguish among the possible statistical processes---

281 let alone biological processes---with any degree of certainty (Volkov et al. 2005), though it

282 is possible that this result differs in marine systems (see Connolly et al. 2014). A more

283 promising way to draw inferences about ecological processes is to evaluate each model's

284 ability to simultaneously explain multiple macroecological patterns, rather than relying on

285 a single pattern like the SAD (McGill 2003, McGill et al. 2006, Newman et al. 2014, Xiao et al.

286 2015). It has also been suggested that examining second-order effects, such as the scale-

287 dependence of macroecological patterns (Blonder et al. 2014) or how the parameters of the 288 distribution change across gradients (Mac Nally et al. 2014), can provide better inference 289 about process from these kinds of pattern. 


\section{Acknowledgments}

291 We thank all of the individuals involved in the collection and provision of the data used in 292 this paper, including the citizen scientists who collect the BBS, $C B C$, and NABC data, the

293 USGS and CWS scientists and managers, the Audubon Society, the North American Butterfly 294 Association, the USDA Forest Service, the Missouri Botanical Garden, and Alwyn H. Gentry. 295 We also thank all of the scientists who published their raw data allowing it to be combined 296 in Baldridge (2013).

\section{References}

298 Alroy, J. 2015. The shape of terrestrial abundance distributions. Science advances 299 1:e1500082.

300 Bache, S. M., and H. Wickham. 2014. Magrittr: A forward-pipe operator for r.

301 Baldridge, E. 2013. Community abundance data.

302 Baldridge, E., D. J. Harris, X. Xiao, and E. P. White. 2016. weecology/sad-comparison: First 303 revision for PeerJ. Zenodo. https://doi.org/10.5281/zenodo.166725.

304 Bayer, M. 2014. Sqlalchemy. The Architecture of Open Source Applications: Elegance, 305 Evolution, and a Few More Fearless Hacks 2.

306 Blonder, B., L. Sloat, B. J. Enquist, and B. McGill. 2014. Separating macroecological pattern 307 and process: Comparing ecological, economic, and geological systems. PloS one 9:e112850. 
308 Boswell, M., and G. Patil. 1971. Chance mechanisms generating the logarithmic series

309 distribution used in the analysis of number of species and individuals. Statistical ecology

310 1:99-130.

311 Bulmer, M. 1974. On fitting the poisson lognormal distribution to species-abundance data.

312 Biometrics:101-110.

313 Burnham, K. P., and D. R. Anderson. 2002. Model selection and multimodel inference: A

314 practical information-theoretic approach. Springer.

315 Clark, R., S. Cox, and G. Laslett. 1999. Generalizations of power-law distributions applicable

316 to sampled fault-trace lengths: Model choice, parameter estimation and caveats.

317 Geophysical Journal International 136:357-372.

318 Cohen, J. E. 1968. Alternate derivations of a species-abundance relation. American

319 naturalist:165-172.

320 Connolly, S. R., M. A. MacNeil, M. J. Caley, N. Knowlton, E. Cripps, M. Hisano, L. M. Thibaut, B.

321 D. Bhattacharya, L. Benedetti-Cecchi, R. E. Brainard, and others. 2014. Commonness and

322 rarity in the marine biosphere. Proceedings of the National Academy of Sciences:8524-

3238529.

324 Edwards, A. M., R. A. Phillips, N. W. Watkins, M. P. Freeman, E. J. Murphy, V. Afanasyev, S. V.

325 Buldyrev, M. G. da Luz, E. P. Raposo, H. E. Stanley, and others. 2007. Revisiting lévy flight

326 search patterns of wandering albatrosses, bumblebees and deer. Nature 449:1044-1048.

327 Engen, S., and R. Lande. 1996. Population dynamic models generating species abundance

328 distributions of the gamma type. Journal of Theoretical Biology 178:325-331. 
329 Fisher, R. A., A. S. Corbet, and C. B. Williams. 1943. The relation between the number of 330 species and the number of individuals in a random sample of an animal population. The 331 Journal of Animal Ecology:42-58.

332 Frank, S. A. 2009. The common patterns of nature. Journal of evolutionary biology $333 \quad 22: 1563-1585$.

334 Frank, S. A. 2011. Measurement scale in maximum entropy models of species abundance. 335 Journal of evolutionary biology 24:485-496.

336 Harte, J. 2011. Maximum entropy and ecology: A theory of abundance, distribution, and 337 energetics. Oxford University Press.

338 Harte, J., T. Zillio, E. Conlisk, and A. Smith. 2008. Maximum entropy and the state-variable 339 approach to macroecology. Ecology 89:2700-2711.

340 Hubbell, S. P. 2001. The unified neutral theory of biodiversity and biogeography (mPB-32).

341 Princeton University Press.

342 Hunter, J. D., and others. 2007. Matplotlib: A 2D graphics environment. Computing in 343 science and engineering 9:90-95.

344 Locey, K. J., and E. P. White. 2013. How species richness and total abundance constrain the 345 distribution of abundance. Ecology letters 16:1177-1185.

346 Mac Nally, R., C. A. McAlpine, H. P. Possingham, and M. Maron. 2014. The control of rank347 abundance distributions by a competitive despotic species. Oecologia 176:849-857. 
348 Matthews, T. J., and R. J. Whittaker. 2014. Fitting and comparing competing models of the

349 species abundance distribution: Assessment and prospect. Frontiers of Biogeography 6.

350 May, R. M. 1975. Patterns of species abundance and diversity. Ecology and evolution of 351 communities:81-120.

352 McGill, B. J. 2003. A test of the unified neutral theory of biodiversity. Nature 422:881-885.

353 McGill, B. J., R. S. Etienne, J. S. Gray, D. Alonso, M. J. Anderson, H. K. Benecha, M. Dornelas, B. 354 J. Enquist, J. L. Green, F. He, and others. 2007. Species abundance distributions: Moving 355 beyond single prediction theories to integration within an ecological framework. Ecology 356 letters 10:995-1015.

357 McGill, B. J., B. A. Maurer, and M. D. Weiser. 2006. Empirical evaluation of neutral theory. 358 Ecology 87:1411-1423.

359 McGill, B., and C. Collins. 2003. A unified theory for macroecology based on spatial patterns 360 of abundance. Evolutionary Ecology Research 5:469-492.

361 McKinney, W., and others. 2010. Data structures for statistical computing in python. Pages 362 51-56 in Proceedings of the 9th python in science conference.

363 Morlon, H., E. P. White, R. S. Etienne, J. L. Green, A. Ostling, D. Alonso, B. J. Enquist, F. He, A. 364 Hurlbert, A. E. Magurran, and others. 2009. Taking species abundance distributions beyond 365 individuals. Ecology Letters 12:488-501.

366 Morris, B. D., and E. P. White. 2013. The ecoData retriever: Improving access to existing 367 ecological data. PloS one 8:e65848. 
368 Newman, E. A., M. E. Harte, N. Lowell, M. Wilber, and J. Harte. 2014. Empirical tests of

369 within-and across-species energetics in a diverse plant community. Ecology 95:2815-2825.

370 Newman, M. E. 2005. Power laws, pareto distributions and zipf's law. Contemporary

371 physics 46:323-351.

372 North American Butterfly Assoc. 2009. NABA butterfly counts: 2009 report. NABA,

373 Morristown, New Jersey, USA.

374 Oliphant, T. E. 2007. Python for scientific computing. Computing in Science \& Engineering 375 9:10-20.

376 Pardieck, K. L., D. J. Ziolkowski Jr, and M.-A. Hudson. 2014. North american breeding bird 377 survey dataset 1966 - 2013, version 2013.0. U.S. Geological Survey, Patuxent Wildlife 378 Research Center.

379 Phillips, O., and J. S. Miller. 2002. Global patterns of plant diversity: Alwyn h. gentry's forest 380 transect data set. Missouri Botanical Garden Press St., Louis, Missouri.

381 Pielou, E. 1975. Ecological diversity. Wiley, New York.

382 Pueyo, S., F. He, and T. Zillio. 2007. The maximum entropy formalism and the idiosyncratic 383 theory of biodiversity. Ecology Letters 10:1017-1028.

384 R Core Team. 2015. R: A language and environment for statistical computing. R Foundation 385 for Statistical Computing, Vienna, Austria.

386 Society, N. A. 2002. The christmas bird count historical results. National Audobon Society, 387 New York, New York, USA. 
388 Sugihara, G. 1980. Minimal community structure: An explanation of species abundance 389 patterns. American naturalist:770-787.

390 Thibault, K. M., S. R. Supp, M. Giffin, E. P. White, and S. M. Ernest. 2011. Species composition

391 and abundance of mammalian communities: Ecological archives e092-201. Ecology 392 92:2316-2316.

393 Tokeshi, M. 1993. Species abundance patterns and community structure. Advances in 394 ecological research 24:111-186.

395 Ulrich, W., M. Ollik, and K. I. Ugland. 2010. A meta-analysis of species-abundance 396 distributions. Oikos 119:1149-1155.

397 USDA Forest Service. 2010. Forest inventory and analysis national core field guide (phase 2 398 and 3). version 4.0. USDA Forest Service, Forest Inventory; Analysis.

399 Van Der Walt, S., S. C. Colbert, and G. Varoquaux. 2011. The numPy array: A structure for 400 efficient numerical computation. Computing in Science \& Engineering 13:22-30.

401 Van Rossum, G., and F. L. Drake. 2011. The python language reference manual. Network 402 Theory Ltd.

403 Volkov, I., J. R. Banavar, F. He, S. P. Hubbell, and A. Maritan. 2005. Density dependence 404 explains tree species abundance and diversity in tropical forests. Nature 438:658-661.

405 Volkov, I., J. R. Banavar, S. P. Hubbell, and A. Maritan. 2003. Neutral theory and relative 406 species abundance in ecology. Nature 424:1035-1037. 
407 White, E. P., B. J. Enquist, and J. L. Green. 2008. On estimating the exponent of power-law 408 frequency distributions. Ecology 89:905-912.

409 White, E. P., K. M. Thibault, and X. Xiao. 2012. Characterizing species abundance 410 distributions across taxa and ecosystems using a simple maximum entropy model. Ecology $41193: 1772-1778$.

412 Wickham, H. 2009. Ggplot2: Elegant graphics for data analysis. Springer-Verlag New York.

413 Wickham, H. 2016. Tidyr: Easily tidy data with 'spread()' and 'gather()' functions.

414 Wickham, H., and R. Francois. 2016. Dplyr: A grammar of data manipulation.

415 Xiao, X., D. J. McGlinn, and E. P. White. 2015. A strong test of the maximum entropy theory 416 of ecology. The American Naturalist 185:E70-E80.

417 Xiao, X., K. Thibault, D. J. Harris, E. Baldridge, and E. White. 2016.

418 Weecology/macroecotools: V0.4.0. Zenodo. http://doi.org/10.5281/zenodo.166721. 